\title{
Article
}

\section{Dependence of Heart Rate Variability Indices on the Mean Heart Rate in Women with Well-Controlled Type 2 Diabetes}

\author{
Adriana Robles-Cabrera ${ }^{1}\left(\mathbb{D}\right.$, José $M$. Torres-Arellano ${ }^{2}\left(\mathbb{D}\right.$, Ruben Fossion $^{3,4}\left(\mathbb{D}\right.$ and Claudia Lerma ${ }^{2, *(\mathbb{D}}$ \\ 1 Biomedical Sciences, National Autonomous University of Mexico, Mexico City 04510, Mexico; \\ adriana.robles@c3.unam.mx \\ 2 Department of Electromechanical Instrumentation, National Institute of Cardiology Ignacio Chávez, \\ Mexico City 14080, Mexico; jose190288@live.com.mx \\ 3 Center for Complexity Sciences (C3), National Autonomous University of Mexico, Mexico City 04510, Mexico; \\ ruben.fossion@nucleares.unam.mx \\ 4 Institute for Nuclear Science, National Autonomous University of Mexico, Mexico City 04510, Mexico \\ * Correspondence: lermag@unam.mx; Tel.: +52-(55)-55732911 (ext. 26202); Fax: +52-(55)-55730926
}

check for

updates

Citation: Robles-Cabrera, A.; Torres-Arellano, J.M.; Fossion, R.; Lerma, C. Dependence of Heart Rate Variability Indices on the Mean Heart Rate in Women with Well-Controlled Type 2 Diabetes. J. Clin. Med. 2021, 10, 4386. https://doi.org/10.3390/ jcm10194386

Academic Editors: Nandu Goswami and Patrick De Boever

Received: 3 July 2021

Accepted: 12 August 2021

Published: 25 September 2021

Publisher's Note: MDPI stays neutral with regard to jurisdictional claims in published maps and institutional affiliations.

Copyright: (c) 2021 by the authors. Licensee MDPI, Basel, Switzerland. This article is an open access article distributed under the terms and conditions of the Creative Commons Attribution (CC BY) license (https:// creativecommons.org/licenses/by/ $4.0 /)$.

\begin{abstract}
Heart rate variability (HRV) is a method used to evaluate the presence of cardiac autonomic neuropathy (CAN) because it is usually attributed to oscillations in cardiac autonomic nerve activity. Recent studies in other pathologies suggest that HRV indices are strongly related to mean heart rate, and this does not depend on autonomic activity only. This study aimed to evaluate the correlation between the mean heart rate and the HRV indices in women patients with well-controlled T2DM and a control group. HRV was evaluated in 19 T2DM women and 44 healthy women during basal supine position and two maneuvers: active standing and rhythmic breathing. Time-domain (SDNN, RMSSD, pNN20) and frequency-domain (LF, HF, LF/HF) indices were obtained. Our results show that meanNN, age, and the maneuvers are the main predictors of most HRV indices, while the diabetic condition was a predictor only for pNN20. Given the known reduced HRV in patients with T2DM, it is clinically important that much of the HRV indices are dependent on heart rate irrespective of the presence of T2DM. Moreover, the multiple regression analyses evidenced the multifactorial etiology of HRV.
\end{abstract}

Keywords: type 2 diabetes mellitus; women; heart rate variability; mean heart rate; orthostatic challenge; rhythmic breathing

\section{Introduction}

Type 2 diabetes mellitus (T2DM) is a metabolic disease characterized by hyperglycemia due to alterations in the production and action of the hormone insulin, which is related to neuropathy and cardiovascular comorbidities [1]. Worldwide, there are more women than men with T2DM, and women have a worse prognosis [2]. The sex dimorphism presented in T2DM is associated with gender-sensitive external (socio-economic and environmental) and internal (genetic, epigenetic, metabolic, hormonal, and behavioral) factors [3]. In 2019, T2DM caused the death of approximately 2.5 million women between 50 and 70 years, reducing life expectancy by around 7 years [4]. T2DM patients have an increase in the hazard ratio for cardiovascular mortality between 60-70 years, which is associated with the development of cardiac autonomic neuropathy or "CAN" [5].

CAN is one of the most prevalent diabetes comorbidities, related to important causes of cardiac death such as lethal arrhythmias, silent myocardial ischemia, and sudden cardiac death [6]. This type of dysautonomia is characterized by sympathovagal imbalance, in which sympathetic activity predominates over parasympathetic activity [7], inducing an increase in heart rate and blood pressure [8] at the systemic level, and generating alterations in the cardiac conduction system and myocardial contractility [9]. Since the 1990s, heart rate variability (HRV) has been a tool widely used to indirectly measure activity from 
the autonomous nervous system (ANS), both from the sympathetic and parasympathetic branches, through its effect on heart rate (HR) [10]. In recent years, some studies have found a close and direct dependence of HRV on average HR in healthy human subjects in rest [11,12] and during cardiovascular reflex testing (the Ewing battery) [13,14], animal models and isolated heart tissue [15]. These studies argue that HRV does not give additional information on the ANS beyond average HR. However, other studies have demonstrated that HRV has prognostic power on morbidity and mortality in patients with cardiovascular and other diseases that is independent of the average HR and therefore should provide valuable extra information on cardiac autonomic regulation [16-20]. Therefore, the relation between HRV and average HR is not well understood in healthy human subjects, and even less so in the presence of pathology.

Although HRV has been studied in many samples of diabetic patients [21-26], the dependence of HRV on the mean heart rate of diabetic patients has not been documented. This study aimed to evaluate the correlation between the mean heart rate and the HRV indices in women patients with well-controlled T2DM and a control group. Multivariate linear regression analyses were applied to corroborate the dependence with other predictor variables such as age and the presence of T2DM.

\section{Materials and Methods}

\subsection{Study Protocol and Patients}

Diabetic patients $(n=19)$ were recruited from the Integral Care Center for Diabetic Patients (CAIPADI) of Instituto Nacional de Ciencias Médicas y Nutrición Salvador Zubirán (INCMNSZ). They were selected using strict inclusion criteria to ensure metabolic and blood pressure control (age 35-60 years, BMI $<30 \mathrm{~kg} / \mathrm{m}^{2}, \mathrm{HbA} 1 \mathrm{c}<6.5 \%$, fasting glucose $<130 \mathrm{mg} / \mathrm{dL}, \mathrm{LDL}<130 \mathrm{mg} / \mathrm{dL}, \mathrm{SBP}<140 \mathrm{mmHg}$, DBP $<90 \mathrm{mmHg}$, with no evidence of diabetic retinopathy or nephropathy diagnosed by a specialist, without any kind of acute or chronic diseases such as infectious, psychiatric, rheumatic, or gastrointestinal illness, among others, without antidepressant, anxiolytic or analgesic treatment). The healthy group $(\mathrm{n}=44)$ were volunteers recruited from the Universidad Nacional Autónoma de México and the Instituto Nacional de Cardiología Ignacio Chávez, with the same inclusion criteria as the diabetic patients, except for fasting glucose $<100 \mathrm{mg} / \mathrm{dL}$. Subjects with electrocardiogram abnormalities, endocrine, cardiovascular, immune, or neurological diseases, or under hormonal treatment were excluded. The diagram of enrollment is shown in Figure 1.

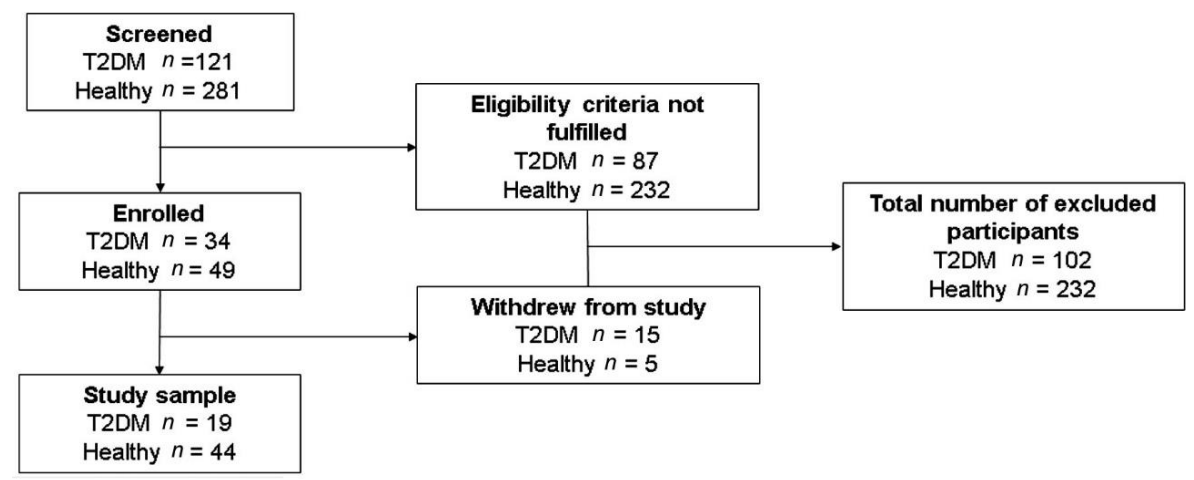

Figure 1. Diagram of enrollment including healthy controls and participants with type 2 diabetes (T2DM).

From each participant a brief medical history was obtained according to the NOM004-SSA3-2012 guideline. Anthropometric measures (height, weight, BMI, blood pressure, and waist circumference), and a blood sample (to obtain glucose, triglycerides, total cholesterol, HDL, LDL, creatinine, uric acid, and ureic nitrogen levels) were obtained. 
Finally, electrocardiogram recordings were obtained to assess HRV with the protocol described below.

\subsection{Ethical Considerations}

This study was conducted with the approval of the Ethics and Research Committee of INCMNSZ, registration number: CONBIOETICA-09-CEI-011-20160627, reference 3102, and the Institutional Ethics Committee of the Instituto Nacional de Cardiología Ignacio Chávez (protocol code 18-1090, approved on 30 October 2018). Each participant was asked to sign the informed consent and the privacy of their data was maintained. None of the participants obtained payment.

\subsection{Materials}

To measure weight and body mass index, an Omron HBF-514C scale was used. Blood pressure was measured with an Omron HEM-712C monitor and height with an InLab S50 portable stadiometer.

\subsection{Blood Sample and Reagents}

The blood samples were analyzed using spectrophotometry (filters 340-620 nm) with Advia 1800 Clinical Chemistry System (Siemens, Munich, Germany). Glucose-hexocinase_3 (GLUH_3, Advia Chemistry, Siemens, Munich, Germany), HbA1c 3M (Advia 1800, Siemens, Munich, Germany), uric acid (Uricase/Peroxidase, Advia 1800, Siemens, Munich, Germany), creatinine (alkaline picrate, Advia 1800, Siemens, Munich, Germany), triglycerides (GPO-PAP method, Advia 1800, Siemens, Munich, Germany), total-, LDL-, and HDLcholesterol (catalase method, Advia 1800, Siemens, Munich, Germany).

\subsection{Electrocardiogram Recording and Beat Detection}

Electrocardiographic recordings were obtained with the Zephyr Bioharness device (Zephyr Performance Systems, Medtronic, Annapolis, MD, USA) using a sampling frequency of $250 \mathrm{~Hz}$.

The study was carried out between 7:00-9:00 a.m. at room temperature between $20-25{ }^{\circ} \mathrm{C}$. The participants were under fasting conditions and it was requested that they not exercise or consume coffee, alcohol, or tobacco the day before. Subjects from the diabetic group were requested to take the medication after the study. The Zephyr Bioharness band was placed on the chest of the participants. The quality of the signal was verified in real time with the IoTool Platform Zephyr Sensors application (SenLab, Slovenia, Balkans). To start the recording protocol, each participant was asked to lie down on a stretcher, remain silent and with closed eyes.

The HRV protocol was conducted under the considerations reported in Laborde et al., 2017 [27] to evaluate tonic (supine) and phasic HRV (active standing and rhythmic breathing), which shows the reactivity or response of the system to two different physiological challenges represented as changes in HRV (supine vs standing or supine vs breathing). The ECG was recorded in supine position (10 $\mathrm{min})$, followed by two consecutive physiological maneuvers of active standing, and rhythmic breathing (at $0.1 \mathrm{~Hz}$ or 6 breaths per minute) of 10 min each (Figure 2).

Active standing is a hemodynamic stimulus in which the drop in blood pressure due to the gravitational force is avoided through an increased sympathetic activity towards the blood vessels (which increases peripheral vascular resistance) combined with a decreased parasympathetic activity (which increases heart rate) $[28,29]$. Rhythmic breathing is a stimulus that increases cardiorespiratory interactions through both the baroreceptors and the chemoreceptors [30]. While the cardiorespiratory interactions during spontaneous breathing usually influence $\mathrm{HRV}$ at around $0.25 \mathrm{~Hz}$, rhythmic breathing at $0.1 \mathrm{~Hz}$ induces a strong cardiorespiratory coupling, and most of the power spectrum energy of the HRV shifts around this low frequency $(0.1 \mathrm{~Hz})[30]$. 
(a)

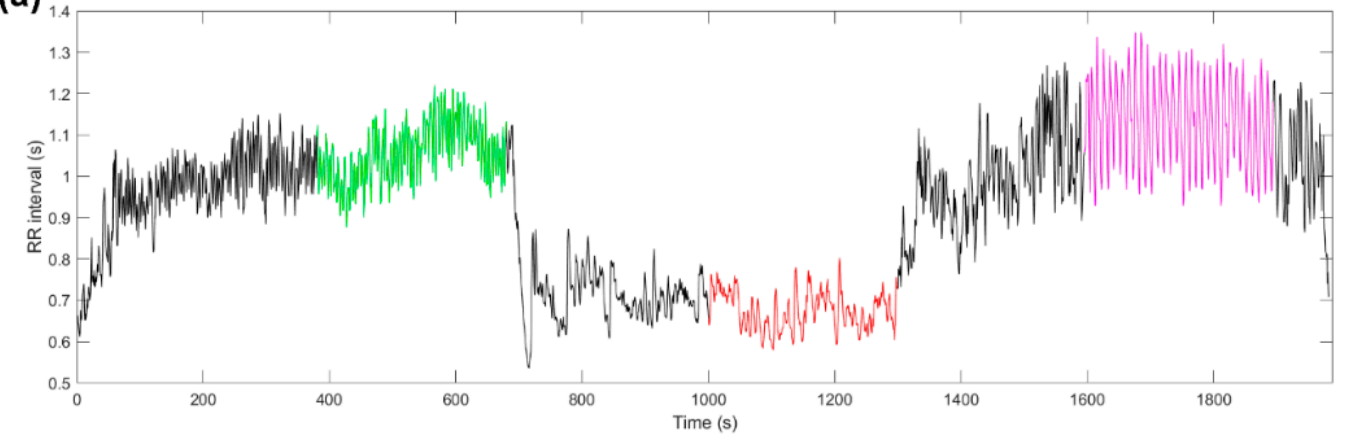

(b)
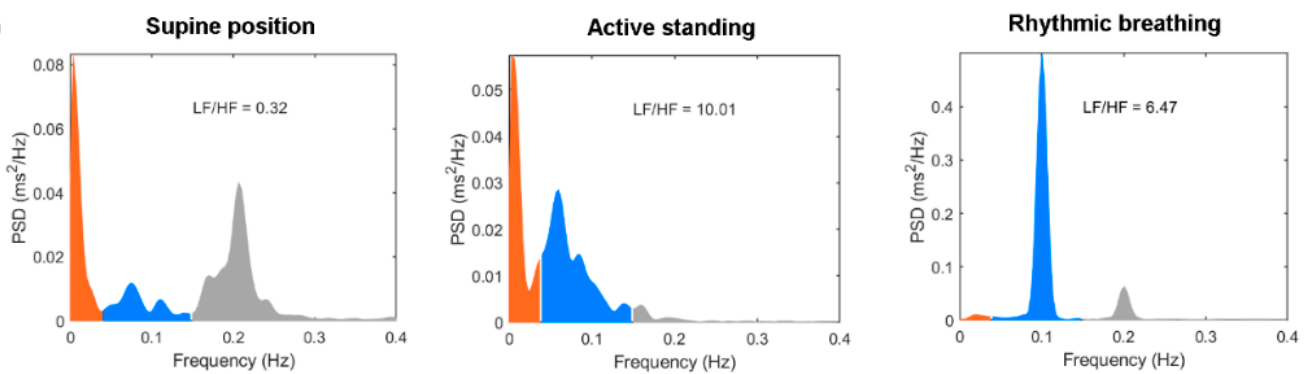

Figure 2. Example of HRV analysis from a healthy subject. (a) Time series obtained from the entire recording protocol with selected 5-min segments that we used in the HRV analysis are indicated with color: supine position (green), active standing (red), and rhythmic breathing (magenta). (b) Power spectrum density (PSD) from the selected segments: very low-frequency (orange), low-frequency (blue), high-frequency (grey), (LF/HF = ratio of low-frequency band power with respect to high frequency band power).

Each ECG recording was transferred to a computer with the program BioHarness log downloader (Medtronic, USA) for further off-line processing and analysis. Identification of the QRS complex of each heartbeat was obtained with a computer program previously validated [31]. Miss-detected heartbeats were identified by visual inspection and corrected manually. The RR interval times series was measured from the period between consecutive QRS complexes. An adaptive filter was applied to the RR intervals to identify RR intervals from ectopic origin and to obtain RR intervals from sinus node origin only (NN intervals or HRV) [32].

\subsection{HRV Analysis}

The correct detection of the RR intervals was first evaluated in the software Processing [31] and the HRV parameters were measured in MATLAB R2020b. After the adaptation time ( $5 \mathrm{~min}$ ), segments of $5 \mathrm{~min}$ were selected in supine position, active standing, and rhythmic breathing (Figure 2a).

For each time series, time-domain parameters such as average value of all $R R$ intervals (MeanNN), standard deviation of all RR intervals (SDNN), square root of the average of the squared differences between adjacent R-R intervals (RMSSD), percentage of successive $R R$ intervals with differences larger than $20 \mathrm{~ms}$ (pNN20) were calculated. To estimate the indices in the frequency domain, each time series was resampled with a linear interpolation of three samples per second and Welch's periodogram method was used to estimate the density of the power spectrum. For the low frequency (LF) band $(0.04$ to $0.15 \mathrm{~Hz})$ and high frequency (HF) band ( 0.15 to $0.4 \mathrm{~Hz}$ ). The spectral power was calculated in normalized units (n.u). Time and frequency domain indices have been associated with autonomic activity. The HF index is reliably related to the control of vagal activity, the LF index reflects both parasympathetic and sympathetic activity [33]. Figure $2 b$ shows a representative example of the power spectral density (PSD) in each analyzed segment. 


\subsection{Statistical Analysis}

The statistical analysis was performed with the Statistical Package for Social Sciences (SPSS) version 25.0 (IBM, Armonk, NY, USA). A $p$-value $<0.05$ was considered statistically significant. Normal distributions were confirmed in all study variables through a Kolmogorov-Smirnov test, except for LF/HF, which was transformed to logarithmic units. The results are described as mean \pm standard deviation and were compared between groups by Student t-tests (two groups) or one-way ANOVA for repeated measures (three or more groups) with post-hoc analysis adjusted by the Bonferroni method. Bivariate correlations were calculated between the meanNN and each HRV index with Pearson's method. Then, linear stepwise multiple regression analyses were performed with each HRV index with a dependent variable and meanNN, maneuver, diabetes mellitus (DM) condition, and age as independent variables. We used the stepwise format of the SPSS program, which "In each step, the independent variable that is not in the equation that has the lowest probability of $\mathrm{F}$ is entered, if that probability is small enough. Variables that are already in the regression equation are eliminated if its probability of $\mathrm{F}$ becomes large enough. The method ends when there are no more variables suitable for inclusion or elimination" according to the SPSS manual.

\section{Results}

\subsection{Antropometric Measures}

Table 1 shows the anthropometric characteristics of the study participants. Compared to the healthy group, the diabetic group was older, had smaller height, larger BMI, and a faster resting average HR. Both groups had similar weight, waist circumference, systolic blood pressure (SBP) and diastolic blood pressure (DBP).

Table 1. Anthropometric characteristics of the study participants.

\begin{tabular}{cccc}
\hline Variable & Healthy Group $(\boldsymbol{N = 4 4 )}$ & Diabetes Group $(\boldsymbol{N}=\mathbf{1 9})$ & $p$-Value \\
\hline Age (years) & $42 \pm 7$ & $52 \pm 7$ & $<0.001$ \\
Height $(\mathrm{m})$ & $1.60 \pm 0.07$ & $1.55 \pm 0.06$ & 0.004 \\
Weight $(\mathrm{kg})$ & $63 \pm 7$ & $63 \pm 7$ & 0.982 \\
BMI (kg/m $)$ & $25 \pm 3$ & $26 \pm 2$ & 0.018 \\
Waist $(\mathrm{cm})$ & $86 \pm 13$ & $86 \pm 7$ & 0.817 \\
Systolic BP (mmHg) & $116 \pm 13$ & $119 \pm 15$ & 0.515 \\
Diastolic BP (mmHg) & $74 \pm 9$ & $73 \pm 6$ & 0.866 \\
Heart rate (bpm) & $61 \pm 9$ & $69 \pm 7$ & $<0.001$ \\
\hline
\end{tabular}

Data are shown as mean \pm standard deviation. $\mathrm{BMI}=$ body mass index; $\mathrm{BP}=$ blood pressure.

\subsection{Metabolic Profile}

The metabolic profile variables were similar between both groups, except for higher serum glucose and uric acid values in the diabetic group compared to the healthy group (Table 2).

Table 2. Metabolic profile variables.

\begin{tabular}{cccc}
\hline Variable & Healthy Group $(\boldsymbol{N}=\mathbf{4 4 )}$ & Diabetes Group $(\boldsymbol{N}=\mathbf{1 9 )}$ & $p$-Value \\
\hline Glucose $(\mathrm{mg} / \mathrm{dL})$ & $79.86 \pm 19.26$ & $99.87 \pm 20.09$ & $<0.001$ \\
Total cholesterol $(\mathrm{mg} / \mathrm{dL})$ & $163.64 \pm 40.40$ & $166.96 \pm 37.33$ & 0.730 \\
HDL $(\mathrm{mg} / \mathrm{dL})$ & $45.74 \pm 15.14$ & $48.82 \pm 11.94$ & 0.380 \\
LDL $(\mathrm{mg} / \mathrm{dL})$ & $101.47 \pm 33.47$ & $105.75 \pm 32.63$ & 0.596 \\
Triglycerides $(\mathrm{mg} / \mathrm{dL})$ & $105.94 \pm 54.39$ & $113.30 \pm 29.68$ & 0.420 \\
Creatinine $(\mathrm{mg} / \mathrm{dL})$ & $0.62 \pm 0.17$ & $0.66 \pm 0.15$ & 0.327 \\
Uric acid $(\mathrm{mg} / \mathrm{dL})$ & $3.82 \pm 0.96$ & $4.66 \pm 1.37$ & 0.034 \\
Ureic nitrogen $(\mathrm{mg} / \mathrm{dL})$ & $11.51 \pm 2.73$ & $13.29 \pm 4.66$ & 0.116 \\
\hline
\end{tabular}


In our diabetes group $(n=19)$, normoglycemic treatment was used in 17 with metformin only, and 2 of them used a diet and exercise regime. The time since diabetes was diagnosed ranged between 0.8 to 10 years, with a median of 2 years.

The participants who smoked totaled 14; 4 in the diabetes group and 10 in the healthy group. None of our participants were consumers of alcohol.

\subsection{HRV Indices between Groups and Maneuvers}

Table 3 shows the HRV indices during baseline and the two maneuvers (supine, active standing, and rhythmic breathing) in both groups. During supine position, the diabetic group had a shorter meanNN (i.e., faster heartbeat) and lower SDNN, RMSSD, and pNN20, indicating less variability in comparison with the healthy group. There were no differences between groups in the spectral indices during the supine position (LF, HF, LF/HF). During active standing, the diabetic group had lower SDNN, LF, and log LF/HF, and higher HF compared to the healthy group. During rhythmic breathing, the diabetic group had lower SDNN, RMSSD, and pNN20 compared to the healthy group.

Table 3. Comparison of HRV indices between groups (healthy and diabetes, $p$ value in the right column) and between maneuvers (supine, active standing, and rhythmic breathing) in the same group ( $\mathrm{a}, \mathrm{b}$ and c markers represent $p$-value).

\begin{tabular}{|c|c|c|c|}
\hline Variable & Healthy Group $(N=44)$ & Diabetes Group $(N=19)$ & $p$-Value \\
\hline \multicolumn{4}{|c|}{ Supine Position } \\
\hline MeanNN (s) & $0.990 \pm 0.135^{\mathrm{a}}$ & $0.920 \pm 0.129^{a, b}$ & 0.018 \\
\hline SDNN (s) & $0.054 \pm 0.027^{\mathrm{a}, \mathrm{b}}$ & $0.041 \pm 0.017^{\mathrm{b}}$ & 0.022 \\
\hline RMSSD (ms) & $47.289 \pm 32.713^{a, b}$ & $26.722 \pm 12.164^{b}$ & 0.001 \\
\hline pNN20 (\%) & $37.277 \pm 17.440^{\mathrm{a}}$ & $21.666 \pm 8.605^{a}$ & $<0.001$ \\
\hline LF (n.u.) & $57.225 \pm 18.837^{\mathrm{a}, \mathrm{b}}$ & $63.060 \pm 14.685^{b}$ & 0.231 \\
\hline HF (n.u.) & $43.019 \pm 18.641^{\mathrm{a}, \mathrm{b}}$ & $36.940 \pm 14.685^{b}$ & 0.203 \\
\hline $\log (\mathrm{LF} / \mathrm{HF})$ & $0.151 \pm 0.379^{\mathrm{a}, \mathrm{b}}$ & $0.257 \pm 0.291^{b}$ & 0.310 \\
\hline \multicolumn{4}{|c|}{ Active Standing } \\
\hline MeanNN (s) & $0.811 \pm 0.104^{\mathrm{c}}$ & $0.822 \pm 0.104^{c}$ & 0.714 \\
\hline SDNN (s) & $0.047 \pm 0.015^{\mathrm{c}}$ & $0.037 \pm 0.012^{\mathrm{c}}$ & 0.008 \\
\hline RMSSD (ms) & $24.354 \pm 11.148^{c}$ & $19.371 \pm 7.536^{c}$ & 0.052 \\
\hline pNN20 (\%) & $23.765 \pm 14.395^{c}$ & $15.378 \pm 9.040^{\mathrm{c}}$ & 0.139 \\
\hline LF (n.u.) & $77.512 \pm 13.298^{c}$ & $70.153 \pm 15.681^{c}$ & 0.034 \\
\hline HF (n.u.) & $22.830 \pm 13.256^{\mathrm{c}}$ & $30.445 \pm 15.858^{c}$ & 0.031 \\
\hline $\log (\mathrm{LF} / \mathrm{HF})$ & $0.612 \pm 0.375^{\mathrm{c}}$ & $0.418 \pm 0.363^{c}$ & 0.037 \\
\hline \multicolumn{4}{|c|}{ Rhythmic Breathing } \\
\hline MeanNN (s) & $1.025 \pm 0.126$ & $1.000 \pm 0.149$ & 0.453 \\
\hline SDNN (s) & $0.098 \pm 0.034$ & $0.068 \pm 0.025$ & $<0.001$ \\
\hline RMSSD (ms) & $72.190 \pm 32.088$ & $49.767 \pm 24.268$ & 0.004 \\
\hline pNN20 (\%) & $32.075 \pm 5.832$ & $25.215 \pm 7.544$ & $<0.001$ \\
\hline LF (n.u.) & $89.443 \pm 5.160$ & $87.828 \pm 7.107$ & 0.273 \\
\hline HF (n.u.) & $10.591 \pm 5.137$ & $12.223 \pm 7.112$ & 0.267 \\
\hline $\log (\mathrm{LF} / \mathrm{HF})$ & $0.979 \pm 0.250$ & $0.913 \pm 0.261$ & 0.302 \\
\hline
\end{tabular}

Data are shown as mean \pm standard deviation. ${ }^{\mathrm{a}} p$-value $<0.05$ supine position vs. active standing (within the same group). ${ }^{\mathrm{b}} p$-value $<0.05$ supine position vs. rhythmic breathing (within the same group). ${ }^{c} p$-value $<0.05$ active standing vs. rhythmic breathing (within the same group).

Comparisons of supine position versus active standing within the healthy group showed the expected acceleration of heart rate (shorter meanNN), decreased variability (lower SDNN, RMSSD, pNN20), increased sympathetic activity (higher LF and log LF/HF) and decreased parasympathetic activity (lower HF). In contrast, the diabetes group responded to active standing with increased heart rate (shorter meanNN) and decreased variability (lower pNN20) but without significant changes in LF, HF, and log LF/HF.

Rhythmic breathing induces more regularity and periodicity in the HR time series; in particular at the specific frequency of $0.1 \mathrm{~Hz}$ the amplitude of the oscillation is maximized, 
which translates into larger variability. In our study, the maneuver of rhythmic breathing induced similar responses in both groups: increasing variability and LF preponderance, because HR follows the same frequency as respiration and $0.1 \mathrm{~Hz}$ falls within the LF band.

\subsection{Correlations between meanNN and HRV Indices}

The correlation analyses were applied combining the sample from the baseline condition (supine position) and each of the two maneuvers (either active standing or rhythmic breathing) to expand the range of meanNN variation (in the active standing maneuver) or HRV variation (in the rhythmic breathing maneuver). When we combined the data from the supine position and active standing to explore the bivariate correlations between each HRV index and meanNN (as independent variable), the healthy group showed the expected response to active standing in all $\mathrm{HRV}$ indices with significant correlations against meanNN (Figures 3 and 4). In contrast, the diabetes group only showed significant correlations with RMSSD and pNN20 (Figure 3).
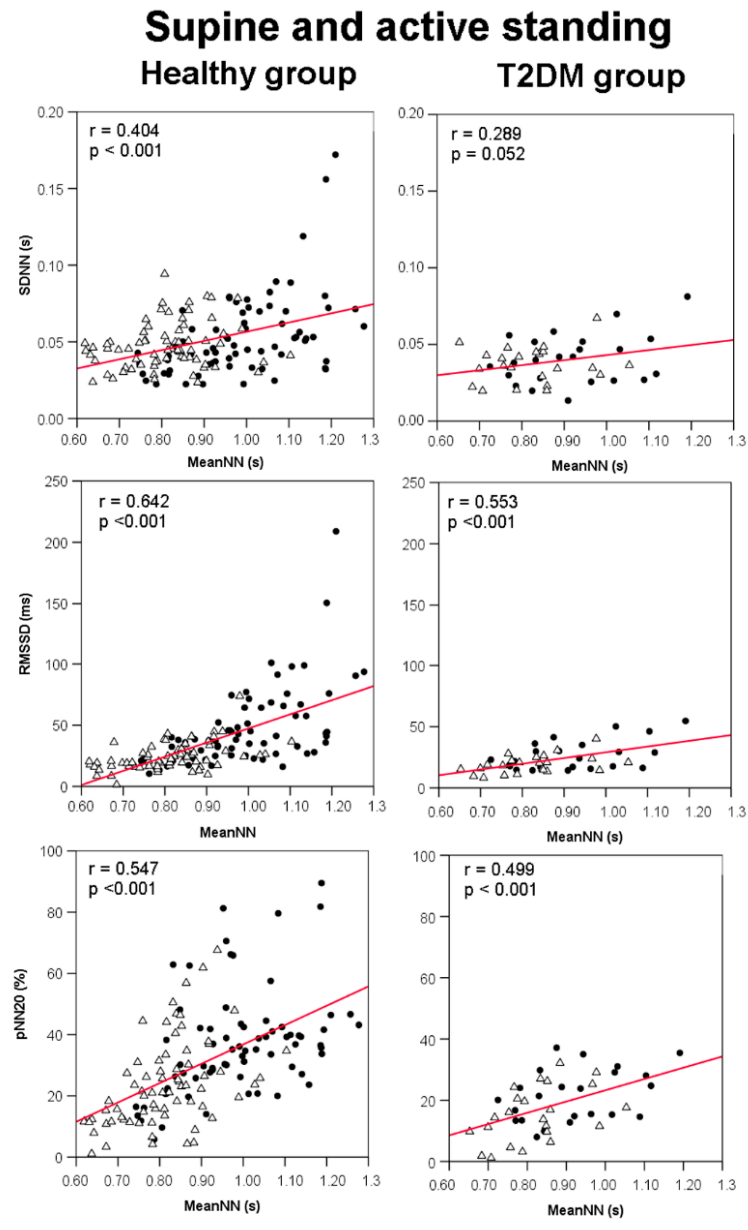

\section{Supine and rhythmic breathing Healthy group}
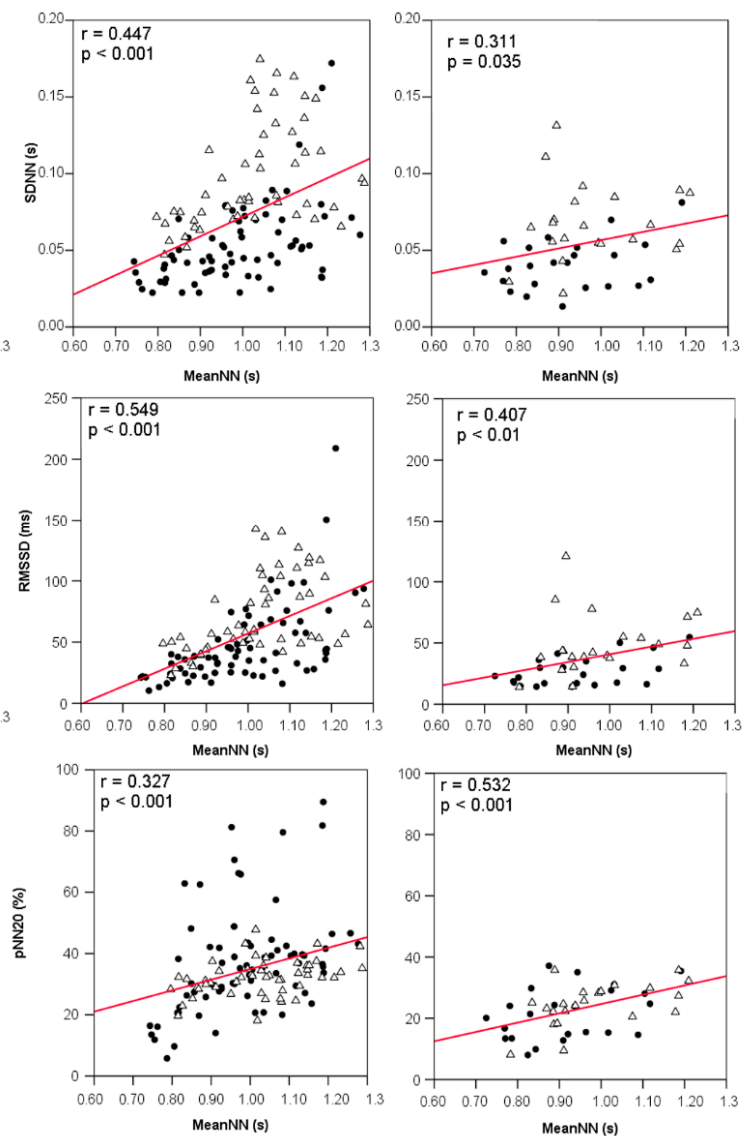

Figure 3. Dispersion plots between time domain HRV indices and meanNN in 44 healthy women (healthy group) and 19 female patients with type-2 diabetes mellitus (T2DM group). The plots on the left panels combine the HRV indices measured during both supine position (filled circles) and active standing (open triangles) of each group ( $N=88$ for the healthy group and $N=38$ for the T2DM group). The plots on the right panels combine the HRV indices measured during both supine position (filled circles) and rhythmic breathing (open triangles) of each group ( $N=88$ for the healthy group and $N=38$ for the T2DM group). MeanNN: mean value of all NN intervals; SDNN: standard deviation of all RR intervals; RMSSD: square root of the average of the squared differences between adjacent R-R intervals; pNN20: percentage of successive RR intervals with differences greater to $20 \mathrm{~ms} ; \mathrm{r}=$ Pearson's correlation coefficient; $p=p$ value. 


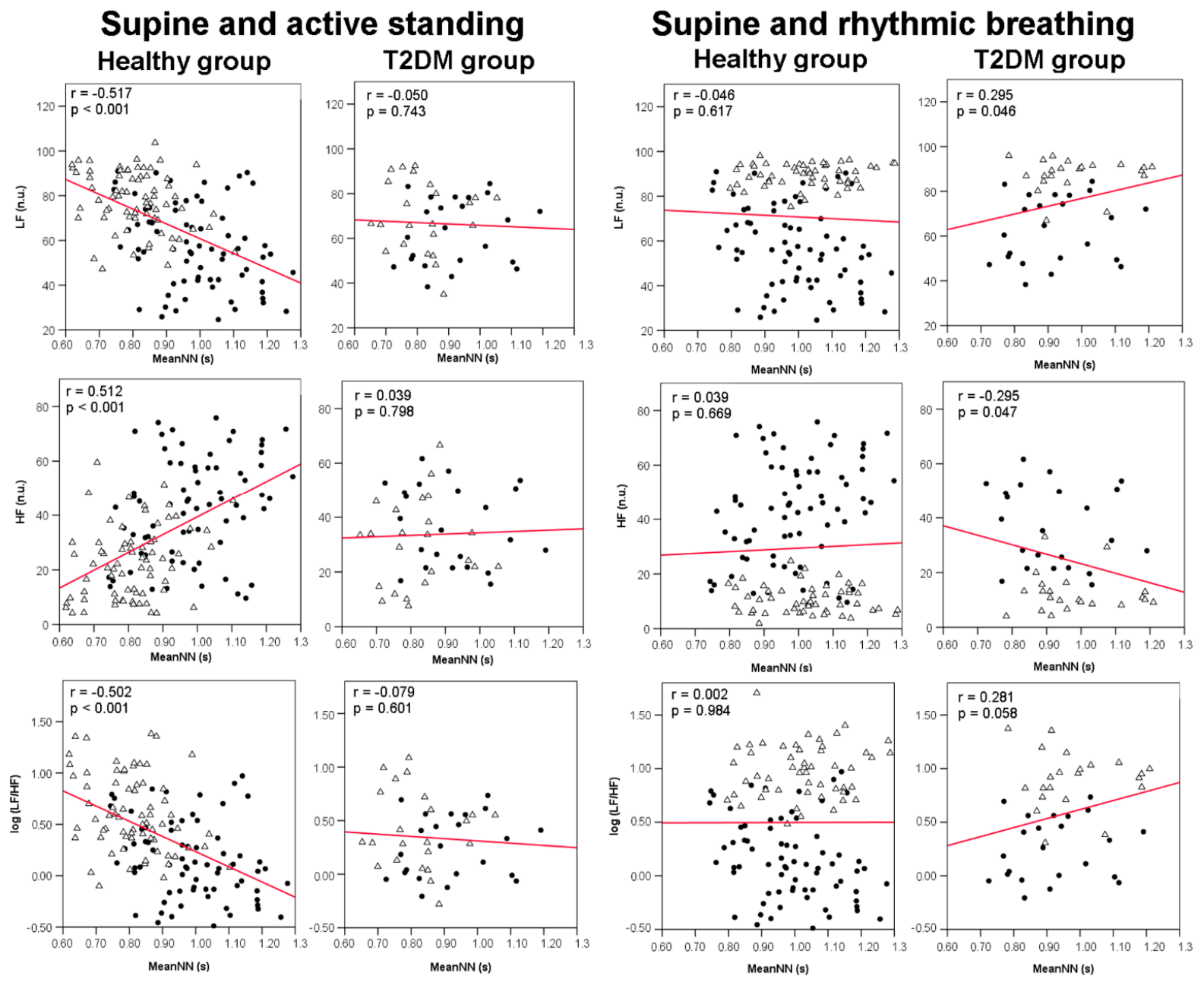

Figure 4. Dispersion plots between frequency domain HRV indices and meanNN in 44 healthy women (healthy group) and 19 female patients with type-2 diabetes mellitus (T2DM group). The plots on the left panels combine the HRV indices measured during both supine position (filled circles) and active standing (open triangles) of each group ( $N=88$ for the healthy group and $N=38$ for the T2DM group). The plots on the right panels combine the HRV indices measured during both supine position (filled circles) and rhythmic breathing (open triangles) of each group ( $N=88$ for the healthy group and $N=38$ for the T2DM group). MeanNN: mean value of all NN intervals; LF: low frequency band index; HF: high frequency band index; log (LF/HF): logarithm of the LF to HF ratio; n.u. = normalized units; $\mathrm{r}=$ Pearson's correlation coefficient; $p=p$ value.

The combined data from supine position and rhythmic breathing showed that both groups have significant positive correlations in time-domain measures, but in the diabetes group the frequency domain parameters (LF and log LF/HF were positive and HF negative) were also significant (Figures 3 and 4 ).

\subsection{Multiple Regression Analysis: Supine and Active Standing}

Multiple linear regression analysis for supine and active standing (Table 4) confirmed that the changes in all HRV indices are associated with meanNN. The other independent factors were age (SDNN and RMSSD), diabetes mellitus condition (pNN20) or the active standing maneuver (LF, HF, and log LF/HF). 
Table 4. Linear stepwise multiple regression analysis with predicted HRV indices and, as independent variables, the meanNN, maneuver, diabetes mellitus (DM) condition, and age. The regression models were applied on the combined samples from supine position and active standing in both groups (healthy and diabetes).

\begin{tabular}{|c|c|c|c|c|}
\hline Variables & Standardized $\beta$ & $\beta($ C.I.95\%) & $p$-Value & $\mathbf{R}^{2}$ \\
\hline \multicolumn{4}{|c|}{ Predicted HRV index: SDNN (ms) } & 0.286 \\
\hline meanNN & 0.356 & $52.123(34.20-70.06)$ & $<0.001$ & \\
\hline Age & -0.374 & $-0.948(-1.26$ to -0.64$)$ & $<0.001$ & \\
\hline Maneuver & & Excluded variable & & \\
\hline DM condition & & Excluded variable & & \\
\hline \multicolumn{4}{|c|}{ Predicted HRV index: RMSSD (ms) } & 0.492 \\
\hline meanNN & 0.585 & $99.558(81.98-117.14)$ & $<0.001$ & \\
\hline Age & -0.339 & $-1.000(-1.31$ to -0.70$)$ & $<0.001$ & \\
\hline Maneuver & & Excluded variable & & \\
\hline DM condition & & Excluded variable & & \\
\hline \multicolumn{4}{|c|}{ Predicted HRV index: pNN20 (\%) } & 0.349 \\
\hline meanNN & 0.510 & $58.317(44.94-71.69)$ & $<0.001$ & \\
\hline DM condition & -0.268 & $-10.289(-14.78$ to -5.80$)$ & $<0.001$ & \\
\hline Maneuver & & Excluded variable & & \\
\hline Age & & Excluded variable & & \\
\hline \multicolumn{4}{|c|}{ Predicted HRV index: LF (n.u.) } & 0.253 \\
\hline meanNN & -0.248 & $-31.338(-50.27$ to -12.41$)$ & 0.001 & \\
\hline Maneuver & 0.331 & $12.064(6.60-17.52)$ & $<0.001$ & \\
\hline DM condition & & Excluded variable & & \\
\hline Age & & Excluded variable & & \\
\hline \multicolumn{4}{|c|}{ Predicted HRV index: HF (n.u.) } & 0.248 \\
\hline meanNN & 0.241 & $30.320(11.43-49.21)$ & 0.002 & \\
\hline Maneuver & -0.331 & $-12.006(-17.46$ to -6.56$)$ & $<0.001$ & \\
\hline DM condition & & Excluded variable & & \\
\hline Age & & Excluded variable & & \\
\hline \multicolumn{4}{|c|}{ Predicted HRV index: $\log (\mathrm{LF} / \mathrm{HF})$} & 0.249 \\
\hline meanNN & -0.239 & $-0.691(-1.12$ to -0.26$)$ & 0.002 & \\
\hline Maneuver & 0.334 & $0.278(0.153-0.403)$ & $<0.001$ & \\
\hline DM condition & & Excluded variable & & \\
\hline Age & & Excluded variable & & \\
\hline
\end{tabular}

\subsection{Multiple Regression Analysis: Supine and Rhythmic Breathing}

In the supine and rhythmic breathing linear regression analysis (Table 5), meanNN and maneuver are positive predictors for time-domain parameters (SDNN, RMSSD, and pNN20); age only predicts SDNN and RMSSD, diabetes condition also predicts pNN20. The frequency-domain parameters are predicted by age and maneuver (LF and log LF/HF positive predictors, HF negative predictors).

Table 5. Linear stepwise multiple regression analysis with predicted HRV indices and, as independent variables, the meanNN, maneuver, diabetes mellitus (DM) condition, and age. The regression models were applied on the combined samples from supine position and rhythmic breathing in both groups (healthy and diabetes).

\begin{tabular}{ccccc}
\hline Variables & Standardized $\beta$ & $\beta$ (C.I.95\%) & $p$-Value & $\mathbf{R}^{\mathbf{2}}$ \\
\hline Predicted HRV index: SDNN (ms) & & & 0.526 \\
meanNN & 0.241 & $62.414(33.56-91.27)$ & $<0.001$ \\
Age & -0.369 & $-1.525(-1.98$ to -1.07$)$ & $<0.001$ \\
Maneuver & 0.492 & $17.451(13.67-21.23)$ & $<0.001$ \\
DM condition & & Excluded variable & \\
\hline
\end{tabular}


Table 5. Cont.

\begin{tabular}{|c|c|c|c|c|}
\hline Variables & Standardized $\beta$ & $\beta($ C.I.95\%) & $p$-Value & $\mathbf{R}^{2}$ \\
\hline \multicolumn{4}{|c|}{ Predicted HRV index: RMSSD (ms) } & 0.510 \\
\hline meanNN & 0.351 & $84.576(57.31-111.84)$ & $<0.001$ & \\
\hline Age & -0.436 & $-1.671(-2.10$ to -1.24$)$ & $<0.001$ & \\
\hline Maneuver & 0.282 & $9.282(5.71-12.86)$ & $<0.001$ & \\
\hline DM condition & & Excluded variable & & \\
\hline \multicolumn{4}{|c|}{ Predicted HRV index: pNN20 (\%) } & 0.265 \\
\hline meanNN & 0.361 & $36.337(22.82-49.86)$ & $<0.001$ & \\
\hline DM condition & -0.316 & $-9.675(-13.75$ to -5.60$)$ & $<0.001$ & \\
\hline Maneuver & -0.163 & $-2.246(-4.08$ to -0.41$)$ & 0.017 & \\
\hline Age & & Excluded variable & & \\
\hline \multicolumn{4}{|c|}{ Predicted HRV index: LF (n.u.) } & 0.551 \\
\hline Age & 0.135 & $0.325(0.08-0.57)$ & 0.010 & \\
\hline Maneuver & 0.736 & 15.206 (13.09-17.32) & $<0.001$ & \\
\hline meanNN & & Excluded variable & & \\
\hline DM condition & & Excluded variable & & \\
\hline \multicolumn{4}{|c|}{ Predicted HRV index: HF (n.u.) } & 0.557 \\
\hline Age & -0.135 & $-0.324(-0.57--0.08)$ & 0.010 & \\
\hline Maneuver & -0.740 & $\begin{array}{l}-15.278(-17.38 \text { to }-13.18) \\
\text { Excluded variable }\end{array}$ & $<0.001$ & \\
\hline DM condition & & Excluded variable & & \\
\hline \multicolumn{4}{|c|}{ Predicted HRV index: $\log (\mathrm{LF} / \mathrm{HF})$} & 0.612 \\
\hline Age & 0.124 & $0.007(0.002-0.013)$ & 0.011 & \\
\hline Maneuver & 0.778 & $0.392(0.34-0.44)$ & $<0.001$ & \\
\hline meanNN & & Excluded variable & & \\
\hline DM condition & & Excluded variable & & \\
\hline
\end{tabular}

$\mathrm{BMI}$ is a variable related to the metabolic condition. We tested the effect of metabolism on multivariate regression models by including BMI as an additional independent variable (Supplementary Material, Tables S1 and S2). All regression models remain similar except for SDNN and pNN20 in the combination of supine position and active standing, where the BMI was included as an independent predictor with a positive slope.

\section{Discussion}

This study tested physiological maneuvers to stimulate hemodynamic compensatory mechanisms (active standing) and the cardio-respiratory coupling (rhythmic breathing) in diabetic and healthy women, to assess the association between meanNN and the HRV indices during these controlled conditions. Our results showed that meanNN, age, and the maneuver are the main predictors of most HRV indices, while the diabetic condition was a predictor only for pNN20 during both maneuvers. Given the known reduced HRV in patients with T2DM, it is clinically important that much of the HRV indices are dependent on heart rate irrespective of the presence of T2DM. Moreover, the regression analyses evidenced the multifactorial etiology of HRV.

There were few anthropometric differences between the evaluated groups. Age, BMI, and resting HR were higher in the diabetes group. Diabetic participants were older because the age at the diagnosis is around 58 years (57.8 \pm 8.7 ) [34], and we tried to find the population with less than 5 years of disease evolution. In our study design, the strict selection criteria for healthy participants precluded the inclusion of older participants because comorbidities are more likely at older age. Higher BMI and resting HR are related to the pathophysiology of this disease [35,36]. BMI is an important risk factor for diabetes because an excess of adipose tissue is related to insulin resistance and low-grade inflammation causing hyperglycemia [37]. However, the BMI was not an independent predictor of most HRV indices in the present study except for SDNN and pNN20 when combining data from supine position and active standing. The elevated resting HR in diabetes has been related to impaired autonomic activity due to increased sympathetic tone related to overweight and neuronal parasympathetic damage [38]. 
Regarding the metabolic profile, the glucose and uric acid blood levels were also higher in the diabetic group. Nevertheless, the diabetic participants are considered as having glycemic control and a normal renal and hepatic function $[39,40]$. High uric acid blood level is a factor which increases the risk of diabetes by $35 \%$ to $48 \%$ compared with non-hyperuricemic populations [41]. Some evidence indicates a bidirectional interaction between the presence of high uric acid and insulin resistance [42] which conditions longterm hyperglycemia.

Heart rate variability (HRV) is a sensitive, non-invasive, and useful biomarker to evaluate autonomic function [43] which has been used to diagnose CAN in type 2 diabetes patients [21-26]. HRV is measured through time and frequency indices. Each is related to the activity of the autonomic nervous system, and higher variability is related to preserved autonomic activity of both sympathetic and parasympathetic branches [33]. The most used temporal parameters are SDNN, which reflects the total power of spectral analysis and the cyclic components of HRV; short term components are related to RMSSD and pNN20 [44]. Some physiological reports describe a close relationship between the activation of the sympathetic branch to LF, and parasympathetic discharge to HF and due to this, an LF/HF ratio was created to evaluate the relation between both subsystems [33].

In our study, we observed that HRV indices were lower (less variability) in the diabetes group than the healthy group in supine position and rhythmic breathing, indicating an autonomic disturbance in the normal parasympathetic resting tone and cardiorespiratory coupling. This coincides with previous findings [24,45]. In healthy subjects, the active standing maneuver increased sympathetic activity, causing an increment of LF and LF/HF indices, but in the diabetes group HF predominated, which could reflect inadequate sympathetic activation [46]. Nevertheless, given the results of the present work about the ubiquitous influence of the heart rate upon HRV indices, caution must be taken when interpreting the HRV indices in comparisons between groups from different populations (e.g., diabetic vs healthy, renal failure vs healthy, young vs old), since a baseline decreased variability in one group cannot be attributed solely to the pathology or comparison factor. Moreover, future studies are required to assess the potential influence of baseline differences in heart rate upon the response of HRV indices to a maneuver such as active standing and rhythmic breathing. Our approach was similar.

Even though the autonomic relationship with HRV has been demonstrated, some studies suggest that HRV is strongly related to average HR independent of other external stimuli [15]. We tested this hypothesis in our sample with Pearson's correlation analysis and linear stepwise multiple regression analysis. In the healthy group, when supine and active standing data were combined to introduce a shift in the set point of HR while increasing its dynamic range, we found that meanNN maintained a significant correlation $(p<0.01)$ with all the time and frequency-domain indices. Meanwhile, in the diabetes group the significant and positive correlations appeared only in RMSSD and pNN20. This apparent loss of HR and HRV correlation in the presence of diabetic disease has not been described and could reflect that in diabetic women, the dynamic behavior of HRV loses adaptability to the orthostatic challenge [47]. In supine and rhythmic breathing, the results of the healthy group show a correlation between HR and HRV only in time-domain, because rhythmic breathing has no effect on mean HR (Table 3), and it influences the HRV indices which reflect statistical variability (time-domain HRV indices). In contrast, rhythmic breathing in the diabetic women decreased mean HR which influenced all the HRV indices except for LF/HF ratio. Rhythmic breathing, also known as paced breathing, at six breaths per minute $(0.1 \mathrm{~Hz})$ tends to cluster the heartbeats in inspiration, modifying the heart rate behavior in a phenomenon known as cardiorespiratory coupling [48]. At $0.1 \mathrm{~Hz}$, the breathing rate increases HRV oscillations at the LF band and the baroreflex sensitivity [49]. The significant correlation of meanNN and HRV indices in the diabetes group and only in the breathing maneuver had not been reported and reflects a conserved cardiorespiratory coupling, which is lost with the progression of diabetes [24]. To date, there is no study that allows us to elucidate the reason for the increase in the correlation between HRV indices in rhythmic 
respiration in T2DM. The evidence supports a decrease in parasympathetic activity and baroreflex sensitivity which is expressed in a cardiorespiratory uncoupling [50] opposite to our findings.

In our work, meanNN is the best and significant predictor of the behavior of HRV indices in supine and active standing, and only in time-domain parameters in rhythmic breathing. This demonstrates the definitive and direct relationship that exists between HRV and meanNN as reported in [15]. However, the maneuver, age, and the presence of diabetes results in independent and significant variables to predict HRV, and they have been also found in other studies in the field. Supine, active standing, and rhythmic breathing are well-known maneuvers proposed by Ewing et al., 1985 [51], that activate fast and consistent autonomic responses in healthy subjects [52], and they are widely used in clinical testing of autonomic function in metabolic [53,54], cardiovascular [55,56], psychological [57], and rheumatic diseases [58]. We expected a significant correlation with HRV indices, demonstrating the existence of cardiac autonomic activity. Normal aging is associated with a decline in cardiovascular system elasticity and compliance, and a diminished autonomic modulation [59]. These effects have been evaluated in baroreflex sensitivity and HRV indices, where both tend to decrease with age and reflect lesser parasympathetic regulation $[60,61]$. The sustained hyperglycemia in type 2 diabetes promotes the damage of the small amyelinic type $C$-fibers of the autonomic nervous systems via oxidative and nitrosative stress, which generates DNA damage, proinflammatory substances release (such as interleukin-1, tumor necrosis factor alpha, and transforming growth factor beta) and a pro-vasoconstriction profile (high levels of endothelin-1 and vascular endothelial growth factor, and a decrease in endothelial nitric oxide synthase production), which results in neuronal toxicity, histological changes, and glial death [62]. In this study, the diabetes mellitus condition results in an independent significant variable only for pNN20, which could be due to the good glycemic control of the sample with diabetes, the short duration of the disease conditions (less than 5 years of progression) and because our group does not present hyperglycemic crisis.

\section{Study Limitations}

Our groups are not age-matched and age is an important predictor for many of the $\mathrm{HRV}$ indices showed in the multiple regression analysis. It was not possible for us to recruit healthy participants of the same age as the participants with diabetes because most of the diabetes participants were over 50 years. In Mexico at this age, most women start to develop obesity, dyslipidemia, hypertension, or other diseases and they must take medication for these alterations. Some of these medications, even hormonal replacement therapy as a treatment for menopause, were exclusion criteria for our work.

For this study, we only have a fasting blood glucose level from healthy women, which confirms short-term control; an $\mathrm{HbA1}$ c value would be adequate to verify long-term glycemic control.

Women with T2DM take metformin or maintain glycemic control with diet and exercise alone, allowing a more homogeneous group, but we did not perform the linear regression analysis to evaluate the correlation that could exist with metformin; for that it is necessary to recruit a larger sample.

The number of participants recruited in the present study was similar to several relevant previous studies $[14,63,64]$. Our sample is relatively small, due to the very strict inclusion and exclusion criteria, which may restrict the generalization of our findings but on the other hand may improve the internal validity of the results.

We did not evaluate the influence of sex-hormones fluctuations related to the sexual female cycle or menopause, which could affect the HRV indices.

In the present study, data from different conditions within the subjects (i.e., supine position, active standing, and rhythmic breathing) were combined. This approach entails a risk of bias for overestimation in the correlation analyses, since combining the samples increases the number of non-independent data points. Nevertheless, the combination of 
data from the resting position and autonomic challenge maneuvers aims to move the set point (meanNN) during the maneuver, which increases the dynamic range of the HRV analysis, as reported in other publications $[63,65,66]$.

The present work evaluated the relationship between mean heart rate and HRV indices of women with T2DM and showed differences between diabetic women and healthy women in the response to a hemodynamic and a cardiorespiratory maneuver. Further studies are required to verify the generalization of our findings in men with T2DM, as well as diabetic patients (both women and men) with other relevant clinical conditions such as lack of metabolic control, other comorbidities including hypertension, the use of some medications that impact the HR and HRV such as beta-blockers, and a greater duration of the diabetes progression to show if our results are consistent.

\section{Conclusions}

This work shows clear differences of HRV indices from T2DM women with good metabolic control when compared to healthy women during supine position, with slightly faster heart rate and lower variability of heart rate, but similar sympatho-vagal balance. In response to active standing, T2DM women reached a lower sympatho-vagal balance, either by a smaller sympathetic activation or not enough parasympathetic withdrawal. Even though T2DM women reached cardiorespiratory coupling with the rhythmic breathing maneuver, they kept a lower variability of heart rate compared to healthy women.

Bivariate correlations analysis between meanNN and HRV indices showed less significant correlations in the diabetes group during active standing, and more significant correlations during rhythmic breathing. This suggests decreased adaptability of the HRV dynamics in diabetic women.

Furthermore, linear regression models demonstrated that meanNN is an independent predictor of most HRV indices, indicating that interpretation of HRV analysis in diabetic women should always consider the contribution of mean heart rate.

Supplementary Materials: The following are available online at https:/ /www.mdpi.com/article/10 $.3390 / \mathrm{jcm} 10194386 / \mathrm{s} 1$, Table S1: Linear stepwise multiple regression analysis with predicted HRV indices and as independent variables the meanNN, maneuver, diabetes mellitus (DM) condition, age, and BMI. The regression models were applied on the combined samples from supine position and active standing, Table S2: Linear stepwise multiple regression analysis with predicted HRV indices and as independent variables the meanNN, maneuver, diabetes mellitus (DM) condition, age, BMI, glucose, and uric acid. The regression models were applied on the combined samples from supine position and rhythmic breathing,

Author Contributions: Conceptualization, C.L.; methodology, C.L. and A.R.-C.; software, C.L., J.M.T.-A., and A.R.-C.; validation, C.L., R.F. and A.R.-C.; formal analysis, C.L., J.M.T.-A. and A.R.-C.; investigation, C.L., R.F. and A.R.-C.; resources, A.R.-C.; data curation, C.L. and A.R.-C.; writingoriginal draft preparation, C.L., J.M.T.-A. and A.R.-C.; writing-review and editing, C.L., R.F. and A.R.-C.; visualization, C.L. and A.R.-C.; supervision, C.L. and R.F.; project administration, C.L. and R.F.; funding acquisition, C.L. and R.F. All authors have read and agreed to the published version of the manuscript.

Funding: This study was supported by CONACYT FORDECYT-PRONACES with grants 610285/ 2020 and 263377/2020, and by the Dirección General de Asuntos del Personal Académico (DGAPA) from the Universidad Nacional Autónoma de México (UNAM) with grant PAPIIT IN110321.

Institutional Review Board Statement: The study was conducted according to the guidelines of the Declaration of Helsinki and approved by the Ethics Committee and Research Committee of Instituto Nacional de Ciencias Médicas y Nutrición "Salvador Zubirán" (INCMNSZ) (protocol code reference 3102, approved in September 2019), as well as by the Institutional Ethics Committee of the Instituto Nacional de Cardiología Ignacio Chávez (protocol code 18-1090, approved on 30 October 2018).

Informed Consent Statement: Written informed consent was obtained from all subjects involved in the study. 
Data Availability Statement: The data presented in this study are available on request from the corresponding author.

\begin{abstract}
Acknowledgments: Adriana Robles Cabrera is a doctoral student from the Programa de Doctorado en Ciencias Biomédicas, Universidad Nacional Autónoma de México (UNAM) and has received CONACYT fellowship 678283. We greatly acknowledge the support in patient recruitment from Cristina García Ulloa, Humberto Del Valle Ramírez and Arturo Flores Gonzalez from the Group of Study CAIPaDi from Instituto Nacional de Ciencias Médicas y Nutrición "Salvador Zubirán". We also appreciate the support of Iván Pérez-Díaz, who conducted the research protocol in Instituto Nacional de Ciencias Médicas y Nutrición "Salvador Zubirán”. We are grateful to QC. Ana Rosalía Lira Reyes (laboratory head), QFB. Emma Viveros Olguín (analyst) and QFB. Jesús Méndez del Ángel (analyst) from Instituto Nacional de Ciencias Médicas y Nutrición “Salvador Zubirán” and David Lara Villalón and work team (especially Deny Marín) from Hospital Juárez de México, to process the samples. We want to thank Michael C. Jeziorski from the Instituto de Neurobiología, UNAM, for his support in the development of the paper.
\end{abstract}

Conflicts of Interest: The authors declare no conflict of interest. The funders had no role in the design of the study; in the collection, analyses, or interpretation of data; in the writing of the manuscript, or in the decision to publish the results.

\title{
References
}

1. Petersmann, A.; Müller-Wieland, D.; Müller, U.A.; Landgraf, R.; Nauck, M.; Freckmann, G.; Heinemann, L.; Schleicher, E. Definition, Classification and Diagnosis of Diabetes Mellitus. Exp. Clin. Endocrinol. Diabetes 2019, 127, S1-S7. [CrossRef] [PubMed]

2. Wild, S.; Roglic, G.; Green, A.; Sicree, R.; King, H. Global Prevalence of Diabetes: Estimates for the year 2000 and projections for 2030. Diabetes Care 2004, 27, 1047-1053. [CrossRef] [PubMed]

3. Gale, E.A.M.; Gillespie, K.M. Diabetes and gender. Diabetologia 2001, 44, 3-15. [CrossRef] [PubMed]

4. International Diabetes Federation, I. Mortality related to Diabetes. In IDF Diabetes Atlas; International Diabetes Federation: Brussels, Belgium, 2019; p. 54.

5. Spallone, V. Update on the Impact, Diagnosis and Management of Cardiovascular Autonomic Neuropathy in Diabetes: What Is Defined, What Is New, and What Is Unmet. Diabetes Metab. J. 2019, 43, 3-30. [CrossRef]

6. Pop-Busui, R.; Braffett, B.H.; Zinman, B.; Martin, C.; White, N.H.; Herman, W.H.; Genuth, S.; Gubitosi-Klug, R. Cardiovascular Autonomic Neuropathy and Cardiovascular Outcomes in the Diabetes Control and Complications Trial/Epidemiology of Diabetes Interventions and Complications (DCCT/EDIC) Study. Diabetes Care 2017, 40, 94-100. [CrossRef]

7. Freeman, R. Diabetic autonomic neuropathy. In Handbook of Clinical Neurology; Elsevier: Amsterdam, The Netherlands, 2014; pp. 63-79.

8. Cabezas-Cerrato, J.; Hermida, R.C.; Cabezas-Agrícola, J.M.; Ayala, D.E. Cardiac Autonomic Neuropathy, Estimated Cardiovascular Risk, and Circadian Blood Pressure Pattern in Diabetes Mellitus. Chronobiol. Int. 2009, 26, 942-957. [CrossRef]

9. Balcığlu, A.S. Diabetes and cardiac autonomic neuropathy: Clinical manifestations, cardiovascular consequences, diagnosis and treatment. World J. Diabetes 2015, 6, 80. [CrossRef]

10. Shaffer, F.; Ginsberg, J.P. An Overview of Heart Rate Variability Metrics and Norms. Front. Public Health 2017, 5, 258. [CrossRef]

11. Sacha, J. Why should one normalize heart rate variability with respect to average heart rate. Front. Physiol. 2013, 4, 306. [CrossRef]

12. Sacha, J. Heart rate contribution to the clinical value of heart rate variability. Kardiol. Pol. 2014, 72, 919-924. [CrossRef]

13. Lefrandt, J.D.; Smit, A.J.; Zeebregts, C.J.; Gans, R.O.B.; Hoogenberg, K.H. Autonomic Dysfunction in Diabetes: A Consequence of Cardiovascular Damage. Curr. Diabetes Rev. 2010, 6, 348-358. [CrossRef] [PubMed]

14. Macartney, M.J.; McLennan, P.L.; Peoples, G.E. Heart rate variability during cardiovascular reflex testing: The importance of underlying heart rate. J. Basic Clin. Physiol. Pharmacol. 2021, 32, 145-153. [CrossRef]

15. Monfredi, O.; Lyashkov, A.E.; Johnsen, A.-B.; Inada, S.; Schneider, H.; Wang, R.; Nirmalan, M.; Wisloff, U.; Maltsev, V.A.; Lakatta, E.G.; et al. Biophysical Characterization of the Underappreciated and Important Relationship Between Heart Rate Variability and Heart Rate. Hypertension 2014, 64, 1334-1343. [CrossRef]

16. Stauss, H.M. Heart Rate Variability: Just a Surrogate for Mean Heart Rate? Hypertension 2014, 64, 1184-1186. [CrossRef] [PubMed]

17. Van Hoogenhuyze, D.; Weinstein, N.; Martin, G.J.; Weiss, J.S.; Schaad, J.W.; Sahyouni, X.N.; Fintel, D.; Remme, W.J.; Singer, D.H. Reproducibility and relation to mean heart rate of heart rate variability in normal subjects and in patients with congestive heart failure secondary to coronary artery disease. Am. J. Cardiol. 1991, 68, 1668-1676. [CrossRef]

18. Sacha, J.; Barabach, S.; Statkiewicz-Barabach, G.; Sacha, K.; Müller, A.; Piskorski, J.; Barthel, P.; Schmidt, G. How to select patients who will not benefit from ICD therapy by using heart rate and its variability? Int. J. Cardiol. 2013, 168, 1655-1658. [CrossRef] [PubMed]

19. Hayano, J.; Sakakibara, Y.; Yamada, A.; Yamada, M.; Mukai, S.; Fujinami, T.; Yokoyama, K.; Watanabe, Y.; Takata, K. Accuracy of assessment of cardiac vagal tone by heart rate variability in normal subjects. Am. J. Cardiol. 1991, 67, 199-204. [CrossRef]

20. Tsuji, H.; Venditti, F.J.; Manders, E.S.; Evans, J.C.; Larson, M.G.; Feldman, C.L.; Levy, D. Reduced heart rate variability and mortality risk in an elderly cohort. The Framingham Heart Study. Circulation 1994, 90, 878-883. [CrossRef] [PubMed] 
21. Michel-Chávez, A.; Estañol, B.; Gien-López, J.A.; Robles-Cabrera, A.; Huitrado-Duarte, M.E.; Moreno-Morales, R.; BecerraLuna, B. Heart Rate and Systolic Blood Pressure Variability on Recently Diagnosed Diabetics. Arq. Bras. Cardiol. 2015, 105, 276-284. [CrossRef]

22. Hoshi, R.A.; Santos, I.S.; Dantas, E.M.; Andreão, R.V.; Schmidt, M.I.; Duncan, B.B.; Mill, J.G.; Lotufo, P.A.; Bensenor, I. Decreased heart rate variability as a predictor for diabetes-A prospective study of the Brazilian longitudinal study of adult health. Diabetes Metab. Res. Rev. 2019, 35, e3175. [CrossRef]

23. Coopmans, C.; Zhou, T.L.; Henry, R.M.A.; Heijman, J.; Schaper, N.C.; Koster, A.; Schram, M.T.; van der Kallen, C.J.H.; Wesselius, A.; den Engelsman, R.J.A.; et al. Both Prediabetes and Type 2 Diabetes Are Associated With Lower Heart Rate Variability: The Maastricht Study. Diabetes Care 2020, 43, 1126-1133. [CrossRef]

24. Rivera, A.L.; Estañol, B.; Fossion, R.; Toledo-Roy, J.C.; Callejas-Rojas, J.A.; Gien-López, J.A.; Delgado-García, G.R.; Frank, A. Loss of Breathing Modulation of Heart Rate Variability in Patients with Recent and Long Standing Diabetes Mellitus Type II. PLoS ONE 2016, 11, e0165904. [CrossRef]

25. Benichou, T.; Pereira, B.; Mermillod, M.; Tauveron, I.; Pfabigan, D.; Maqdasy, S.; Dutheil, F. Heart rate variability in type 2 diabetes mellitus: A systematic review and meta-analysis. PLoS ONE 2018, 13, e0195166. [CrossRef] [PubMed]

26. Shah, A.S.; El ghormli, L.; Vajravelu, M.E.; Bacha, F.; Farrell, R.M.; Gidding, S.S.; Levitt Katz, L.E.; Tryggestad, J.B.; White, N.H.; Urbina, E.M. Heart Rate Variability and Cardiac Autonomic Dysfunction: Prevalence, Risk Factors, and Relationship to Arterial Stiffness in the Treatment Options for Type 2 Diabetes in Adolescents and Youth (TODAY) Study. Diabetes Care 2019, 42, 2143-2150. [CrossRef]

27. Laborde, S.; Mosley, E.; Thayer, J.F. Heart Rate Variability and Cardiac Vagal Tone in Psychophysiological ResearchRecommendations for Experiment Planning, Data Analysis, and Data Reporting. Front. Psychol. 2017, 8, 213. [CrossRef] [PubMed]

28. Hilz, M.J.; Dütsch, M. Quantitative studies of autonomic function. Muscle Nerve 2006, 33, 6-20. [CrossRef] [PubMed]

29. Gerritsen, J.; TenVoorde, B.J.; Dekker, J.M.; Kingma, R.; Kostense, P.J.; Bouter, L.M.; Heethaar, R.M. Measures of cardiovascular autonomic nervous function: Agreement, reproducibility, and reference values in middle age and elderly subjects. Diabetologia 2003, 46, 330-338. [CrossRef] [PubMed]

30. Elstad, M.; O'Callaghan, E.L.; Smith, A.J.; Ben-Tal, A.; Ramchandra, R. Cardiorespiratory interactions in humans and animals: Rhythms for life. Am. J. Physiol. Circ. Physiol. 2018, 315, H6-H17. [CrossRef]

31. Figuerola, S.; Quintanar, E.; Lerma, C. Development and validation of a graphical user interface for assessment of cardiorespiratory coupling. Proc. Congr. Int. Ing. Electrón. Mem. Electro 2019, 41, 148-153.

32. Wessel, N.; Voss, A.; Malberg, H.; Ziehmann, C.; Voss, H.U.; Schirdewan, A.; Meyerfeldt, U.; Kurths, J. Nonlinear analysis of complex phenomena in cardiological data. Herzschrittmachertherapie Elektrophysiologie 2000, 11, 159-173. [CrossRef]

33. Malik, M.; Bigger, J.T.; Camm, A.J.; Kleiger, R.E.; Malliani, A.; Moss, A.J.; Schwartz, P.J. Heart rate variability. Standards of measurement, physiological interpretation, and clinical use. Task Force of the European Society of Cardiology and the North American Society of Pacing and Electrophysiology. Eur. Heart J. 1996, 17, 354-381. [CrossRef]

34. Zoungas, S.; Woodward, M.; Li, Q.; Cooper, M.E.; Hamet, P.; Harrap, S.; Heller, S.; Marre, M.; Patel, A.; Poulter, N.; et al. Impact of age, age at diagnosis and duration of diabetes on the risk of macrovascular and microvascular complications and death in type 2 Diabetes. Diabetologia 2014, 57, 2465-2474. [CrossRef]

35. Bays, H.E.; Chapman, R.H.; Grandy, S. The relationship of body mass index to diabetes mellitus, hypertension and dyslipidaemia: Comparison of data from two national surveys. Int. J. Clin. Pract. 2007, 61, 737-747. [CrossRef] [PubMed]

36. Xu, C.; Zhong, J.; Zhu, H.; Hu, R.; Fang, L.; Wang, M.; Zhang, J.; Guo, Y.; Bian, Z.; Chen, Z.; et al. Independent and interactive associations of heart rate and body mass index or blood pressure with type 2 diabetes mellitus incidence: A prospective cohort study. J. Diabetes Investig. 2019, 10, 1068-1074. [CrossRef] [PubMed]

37. Narayan, K.M.V.; Boyle, J.P.; Thompson, T.J.; Gregg, E.W.; Williamson, D.F. Effect of BMI on Lifetime Risk for Diabetes in the U.S Diabetes Care 2007, 30, 1562-1566. [CrossRef] [PubMed]

38. Aune, D.; Hartaigh, B.ó; Vatten, L.J. Resting heart rate and the risk of type 2 diabetes: A systematic review and dose-response meta-analysis of cohort studies. Nutr. Metab. Cardiovasc. Dis. 2015, 25, 526-534. [CrossRef]

39. Casiglia, E.; Tikhonoff, V.; Virdis, A.; Masi, S.; Barbagallo, C.M.; Bombelli, M.; Bruno, B.; Cicero, A.F.G.; Cirillo, M.; Cirillo, P.; et al. Serum uric acid and fatal myocardial infarction: Detection of prognostic cut-off values: The URRAH (Uric Acid Right for Heart Health) study. J. Hypertens. 2020, 38, 412-419. [CrossRef] [PubMed]

40. Kohnert, K.-D. Utility of different glycemic control metrics for optimizing management of Diabetes. World J. Diabetes 2015, 6, 17. [CrossRef]

41. Liu, J.; Tao, L.; Zhao, Z.; Mu, Y.; Zou, D.; Zhang, J.; Guo, X. Two-Year Changes in Hyperuricemia and Risk of Diabetes: A Five-Year Prospective Cohort Study. J. Diabetes Res. 2018, 2018, 6905720. [CrossRef] [PubMed]

42. Li, C.; Hsieh, M.-C.; Chang, S.-J. Metabolic syndrome, diabetes, and hyperuricemia. Curr. Opin. Rheumatol. 2013, 25, $210-216$. [CrossRef] [PubMed]

43. Sassi, R.; Cerutti, S.; Lombardi, F.; Malik, M.; Huikuri, H.V.; Peng, C.-K.; Schmidt, G.; Yamamoto, Y.; Gorenek, B.; Lip, G.Y.H.; et al. Advances in heart rate variability signal analysis: Joint position statement by the e-Cardiology ESC Working Group and the European Heart Rhythm Association co-endorsed by the Asia Pacific Heart Rhythm Society. Europace 2015, 17, 1341-1353. [CrossRef] [PubMed] 
44. Kurths, J.; Voss, A.; Saparin, P.; Witt, A.; Kleiner, H.J.; Wessel, N. Quantitative analysis of heart rate variability. Chaos Interdiscip. J. Nonlinear Sci. 1995, 5, 88-94. [CrossRef]

45. Yamasaki, Y.; Ueda, N.; Kishimoto, M.; Tani, A.; Ishida, Y.; Kawamori, R.; Kamada, T. Assessment of early stage autonomic nerve dysfunction in diabetic subjects-application of power spectral analysis of heart rate variability. Diabetes Res. 1991, 17, 73-80. [PubMed]

46. Howorka, K.; Pumprla, J.; Jirkovska, A.; Lacigova, S.; Nolan, J. Modified orthostatic load for spectral analysis of short-term heart rate variability improves the sensitivity of autonomic dysfunction assessment. J. Diabetes Complicat. 2010, $24,48-54$. [CrossRef] [PubMed]

47. Ernst, G. Heart-Rate Variability-More than Heart Beats? Front. Public Health 2017, 5, 240. [CrossRef] [PubMed]

48. Dick, T.E.; Hsieh, Y.-H.; Dhingra, R.R.; Baekey, D.M.; Galán, R.F.; Wehrwein, E.; Morris, K.F. Cardiorespiratory Coupling. Prog. Brain Res. 2014, 209, 191-205. [CrossRef] [PubMed]

49. Russo, M.A.; Santarelli, D.M.; O’Rourke, D. The physiological effects of slow breathing in the healthy human. Breathe 2017, 13, 298-309. [CrossRef]

50. Matsutani, D.; Sakamoto, M.; Iuchi, H.; Minato, S.; Suzuki, H.; Kayama, Y.; Takeda, N.; Horiuchi, R.; Utsunomiya, K. Glycemic variability in continuous glucose monitoring is inversely associated with baroreflex sensitivity in type 2 diabetes: A preliminary report. Cardiovasc. Diabetol. 2018, 17, 36. [CrossRef] [PubMed]

51. Ewing, D.J.; Martyn, C.N.; Young, R.J.; Clarke, B.F. The Value of Cardiovascular Autonomic Function Tests: 10 Years Experience in Diabetes. Diabetes Care 1985, 8, 491-498. [CrossRef] [PubMed]

52. Vinik, A.I.; Maser, R.E.; Mitchell, B.D.; Freeman, R. Diabetic Autonomic Neuropathy. Diabetes Care 2003, 26, 1553-1579. [CrossRef] [PubMed]

53. Eleftheriadou, A.; Williams, S.; Nevitt, S.; Brown, E.; Roylance, R.; Wilding, J.P.H.; Cuthbertson, D.J.; Alam, U. The prevalence of cardiac autonomic neuropathy in prediabetes: A systematic review. Diabetologia 2021, 64, 288-303. [CrossRef] [PubMed]

54. Stein, P.K.; Barzilay, J.I.; Domitrovich, P.P.; Chaves, P.M.; Gottdiener, J.S.; Heckbert, S.R.; Kronmal, R.A. The relationship of heart rate and heart rate variability to non-diabetic fasting glucose levels and the metabolic syndrome: The Cardiovascular Health Study. Diabet. Med. 2007, 24, 855-863. [CrossRef] [PubMed]

55. Thayer, J.F.; Yamamoto, S.S.; Brosschot, J.F. The relationship of autonomic imbalance, heart rate variability and cardiovascular disease risk factors. Int. J. Cardiol. 2010, 141, 122-131. [CrossRef] [PubMed]

56. Solaro, N.; Malacarne, M.; Pagani, M.; Lucini, D. Cardiac Baroreflex, HRV, and Statistics: An Interdisciplinary Approach in Hypertension. Front. Physiol. 2019, 10, 478. [CrossRef]

57. Nemeroff, C.B.; Goldschmidt-Clermont, P.J. Heartache and heartbreak-The link between depression and cardiovascular disease. Nat. Rev. Cardiol. 2012, 9, 526-539. [CrossRef] [PubMed]

58. Martinez-Lavin, M.; Hermosillo, A.G.; Rosas, M.; Soto, M.-E. Circadian studies of autonomic nervous balance in patients with fibromyalgia: A heart rate variability analysis. Arthritis Rheum. 1998, 41, 1966-1971. [CrossRef]

59. Barantke, M.; Krauss, T.; Ortak, J.; Lieb, W.; Reppel, M.; Burgdorf, C.; Pramstaller, P.P.; Schunkert, H.; Bonnemeier, H. Effects of Gender and Aging on Differential Autonomic Responses to Orthostatic Maneuvers. J. Cardiovasc. Electrophysiol. 2008, 19, 1296-1303. [CrossRef] [PubMed]

60. Wang, S.Y.; Zhang, L.F.; Wang, X.B.; Cheng, J.H. Age dependency of heart rate variability, blood pressure variability and baroreflex sensitivity. Space Med. Med. Eng. 2000, 13, 318-322.

61. Singh, D.; Vinod, K.; Saxena, S.C.; Deepak, K.K. Spectral evaluation of aging effects on blood pressure and heart rate variations in healthy subjects. J. Med. Eng. Technol. 2006, 30, 145-150. [CrossRef]

62. Agashe, S.; Petak, S. Cardiac Autonomic Neuropathy in Diabetes Mellitus. Methodist Debakey Cardiovasc. J. 2018, 14, 251-256. [CrossRef]

63. Echeverría, J.C.; Infante, O.; Pérez-Grovas, H.; González, H.; José, M.V.; Lerma, C. Effects of Orthostatism and Hemodialysis on Mean Heart Period and Fractal Heart Rate Properties of Chronic Renal Failure Patients. Artif. Organs 2017, 41, 1026-1034. [CrossRef] [PubMed]

64. Torres-Arellano, J.M.; Echeverría, J.C.; Ávila-Vanzzini, N.; Springall, R.; Toledo, A.; Infante, O.; Bojalil, R.; Cossío-Aranda, J.E.; Fajardo, E.; Lerma, C. Cardiac Autonomic Response to Active Standing in Calcific Aortic Valve Stenosis. J. Clin. Med. 2021, 10, 2004. [CrossRef] [PubMed]

65. Lerma, C.; Echeverría, J.C.; Infante, O.; Pérez-Grovas, H.; González-Gómez, H. Sign and magnitude scaling properties of heart rate variability in patients with end-stage renal failure: Are these properties useful to identify pathophysiological adaptations? Chaos Interdiscip. J. Nonlinear Sci. 2017, 27, 093906. [CrossRef]

66. Calderón-Juárez, M.; González-Gómez, G.H.; Echeverría, J.C.; Pérez-Grovas, H.; Lerma, C. Association between Mean Heart Rate and Recurrence Quantification Analysis of Heart Rate Variability in End-Stage Renal Disease. Entropy 2020, $22,114$. [CrossRef] [PubMed] 